\title{
ENSAYO DE UN METODO DE ANALISIS DE VARIABILIDAD FORMAL APLICADO AL TIPO B7 DEL HORIZONTE DEL BRONCE FINAL DE PEÑA NEGRA (850-675 AC)
}

\author{
ALFREDO GONZALEZ PRATS \\ Universidad de Alicante
}

\begin{abstract}
Presentamos un método de análisis de variabilidad tipológica de los cuencos y cazuelas carenados de Peña Negra I, atendiendo a la forma completa («factores primarios») y, en su defecto, a las diferencias en la inclinación, grosor y delineación del borde y modo de desarrollarse la carena («factores secundarios»). La finalidad que se persigue es tanto establecer una pauta tipológica con que operar en el futuro, como lograr detectar alguna variante significativa con posible valor cultural o cronológico. Algunos de estos caracteres tipológicos nos conducen a realizar una rápida panorámica de los posibles precedentes y de sus relaciones con otras áreas peninsulares.

The purpose of this article is to present a method for analysing tipological variation of carinated bowls from Peña Negra I, which will bear in mind the complete outline ("primary factors») or, if this is not available, the differences in delineation, thickness or slope of edge and the variation in the careening («secondary factors»). Our aims are both to find a typological norm for future work and to detect any significant variants with chronological or cultural value. Some of these factors on present in other earlier or contemporary vessels from different areas of the Iberian Peninsula, and these are briefly discussed.
\end{abstract}

\section{INTRODUCCION}

En el ambiente ceramológico de Peña Negra I destaca la producción de unas vasijas de elevado grado de calidad, con pastas bien levigadas y compactas, cuyas superficies hacen gala de un intenso bruñido que les confiere un aspecto metálico. Esta clase de cerámica fina ha sido individualizada como «Grupo B», frente al otro tipo de cerámica grosera que constituye el «Grupo A», dualidad que ya se ha señalado en varias ocasiones (GONZALEZ PRATS, 1977-78, 122; 1979, 163).

Del repertorio tipológico constitutivo del grupo de cerámicas finas, la Forma 7 resulta la más característica, contando además con un alto porcentaje de representatividad dentro del grupo que le confiere una clara entidad, de suma importancia para la caracterización cultural y cronológica de la primera fase de Peña Negra. Las vasijas que integran dicha forma son denominadas en la bibliografía «platos», «cuencos», "cazuelas» o «fuentes» carenadas, sobresaliendo aquellas que presentan una carena alta (SCHUBART, 1971, 171 y 173; MOLINA GONZALEZ, 1977, 15).

La forma es característica de los ambientes del Bronce Final de Portugal, Baja Extremadura, Andalucía y Sudeste, con ligeras matizaciones tecnológicas que afectan tanto al propio acabado de las vasijas como a pequeños detalles morfológicos (LOPEZ 
ROA, 1978, 150 ss.) e incluso al gusto decorativo, que se inscribe en la línea de lo geométrico. Así, mientras en el círculo portugués hay una preferencia por desarrollar los motivos en la superficie externa de los cuencos y en la Baja Andalucía se plasman en su interior -en ambas áreas mediante la técnica del bruñido diferencial-, en la Alta Andalucía gusta representar la temática geométrica mediante el uso de la pintura -monocroma o bicroma- y en el Sudeste los vasos hacen alarde tanto de una minuciosa y perfecta decoración incisa como de la ornamentación pictórica, técnicas ambas que a veces se reúnen en un mismo ejemplar.

\section{METODO DE ANALISIS}

La enorme variabilidad formal observada en Peña Negra en el seno de la formatipo (fenómeno generalizado, por otra parte, en todo el área del Sudeste peninsular) nos indujo a la adopción de un método de análisis susceptible de desglosar y denominar de una forma puntual este amplio repertorio de variantes integradas en el Tipo B7.

Este proceder tendía a un doble objetivo. En primer lugar, llegar a indentificar alguna variable específica con valor cronológico o cultural, puesto que a simple vista las mismas características generales presentaban las vasijas de los estratos inferiores que las de los estratos superiores. En segundo lugar, separar -si los hubiere - distintos grupos de variantes con el fin de analizar la posible procedencia tipológica de los mismos; es decir, intentar rastrear alguna inspiración formal en el repertorio tipológico de períodos y culturas contemporáneos o precedentes. Ello podría traducirse en el mejor encuadre cultural de las manifestaciones materiales existentes en el seno de las comunidades del Bronce Final del yacimiento de Crevillente.

Los criterios que hemos utilizado para la confección, individualización y denominación tipológica de las formas B7 se sitúan en dos órdenes. En el primero se contempla la presencia de unos «factores primarios» que se aprecian cuando el perfil del vaso nos ha llegado completo. En primer lugar, la distinción entre «cuenco» y «cazuela» se realizará observando el carácter «hondo»o «llano» del vaso mediante un índice resultado de la división del diámetro máximo y de la altura o profundidad. Aquellos vasos cuyo índice resulte mayor de 3 se denominarán «cazuelas» y vendrán individualizadas por la letra «A» colocada a continuación de la sigla del tipo genérico (B7A), mientras que los «cuencos» presentarán índices inferiores a 3 y se designarán con la letra «B» $(B 7 B)$. Un segundo factor estima la situación de la línea de carenación en el perfil del vaso, distinguiéndose entre «carena alta» y «carena media», designadas con las cifras 1 y 2 respectivamente.

El tercer factor considera la delineación o carácter del cuerpo inferior de la vasija: convexo (a), rectilíneo o troncocónico (b) y cóncavo (c). Un cuarto factor prevé la existencia de diversas formas de sustentación del vaso: base plana (1), ónfalos (2), base convexa (3) y base anular o con pie diferenciado (4).

A estos factores se les aducirá la indicación del sistema de suspensión empleado:

- perforación vertical en la línea de carena (a).

- perforación horizontal sobre orejeta o mamelón (b).

- sin perforación (c).

- otro sistema (d).

De este modo, una cazuela de carena alta con cuerpo troncocónico, base plana y taladro vertical en la carena tendría la siguiente nomenclatura: B7A1bla.

Con respecto al tamaño de las cazuelas, operaremos con el siguiente módulo atendiendo al diámetro del borde:

$<18 \mathrm{~cm}$ : pequeñas

18-22 cm: medianas

$>22 \mathrm{~cm}$ : grandes (fuentes). 
Pero, desgraciadamente, los ejemplares completos que pueden recuperarse en nuestras excavaciones no son lo suficientemente numerosos como para llevar a cabo estudio analítico, estadístico o comparativo alguno. Debido a este fenómeno, y con el fin de no despreciar la ingente masa de documentación constituida por fragmentos de dichas vasijas, pensamos en un análisis de «factores secundarios» centrado en el estudio de la parte superior de los recipientes en cuestión, cuya variación formal no se podía indicar, además, con el análisis de factores primarios.

El análisis de factores secundarios observa los siguientes caracteres:

Inclinación del borde
A Tumbado $\left(15^{\circ}-44^{\circ}\right)$
B Abierto $\left(45^{\circ}-72^{\circ}\right)$
C Tendencia recta $\left(73^{\circ}-79^{\circ}\right)$
D Recto o vertical $\left(80^{\circ}-98^{\circ}\right)$
E Reentrante $\left(>98^{\circ}\right)$

Tipo de inflexión de la carena

1 Simple poco marcada

2 Simple redondeada

3 Simple acusada

4 Simple de arista viva

5 Hombrera corta redondeada

6 Hombrera corta acusada

7 Hombrera pronunciada redondeada

8 Hombrera pronunciada acusada

9 Ancha redondeada

0 Ancha inclinada

Relación longitud/grosor del borde
A Borde corto delgado
B Borde corto grueso
C Borde largo delgado
D Borde largo grueso

Delineación del borde

1 Rectilíneo

2 Convexo

3 Cóncavo

Inflexión interna

I Con inflexión

$\mathrm{N}$ Sin inflexión

Con semejantes factores se establece un total de 1.200 variantes virtuales de las que, en realidad - al no presentarse bordes con inclinación E- tomaremos 960 en consideración.

Los cinco grupos de variables que anteceden no presentan una misma identificabilidad connotativa entre sus caracteres. Es decir, mientras los caracteres de unos se discriminan mediante determinaciones mensurables, en otros intervienen la delineación geométrica, la proporcionalidad o el simple juego de ausencia-presencia. Así, el primer grupo referente a la inclinación de los bordes expresada en grados sexagesimales incluye una concreta situación en el arco graduado. Para la identificación espacial de cada va- 


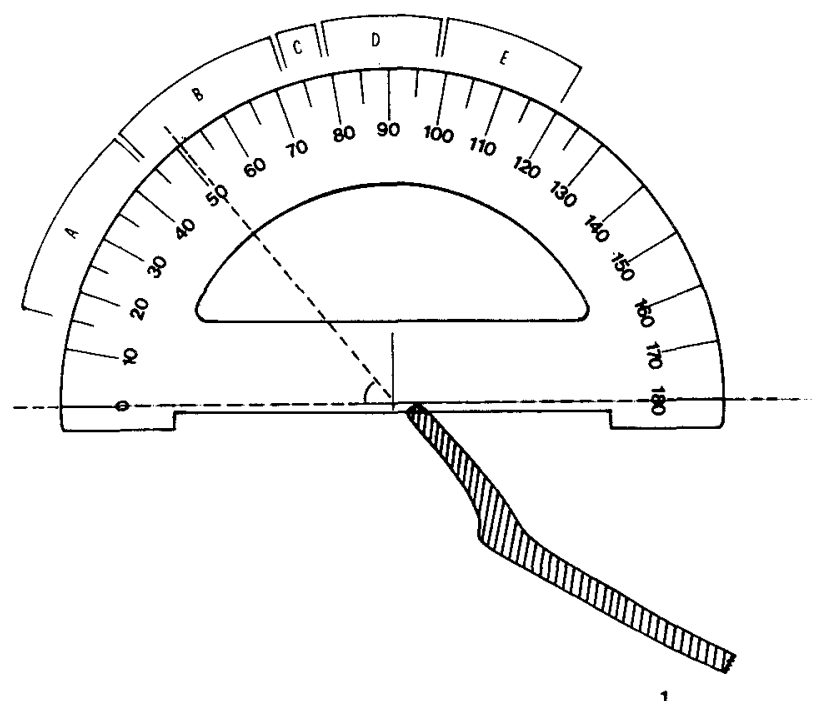

3
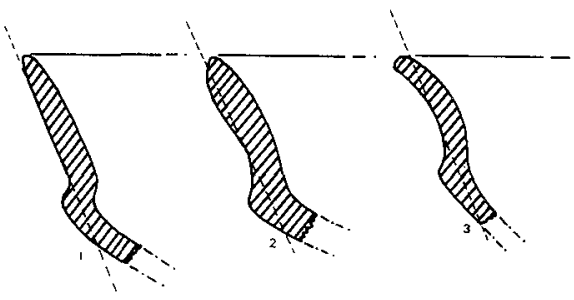
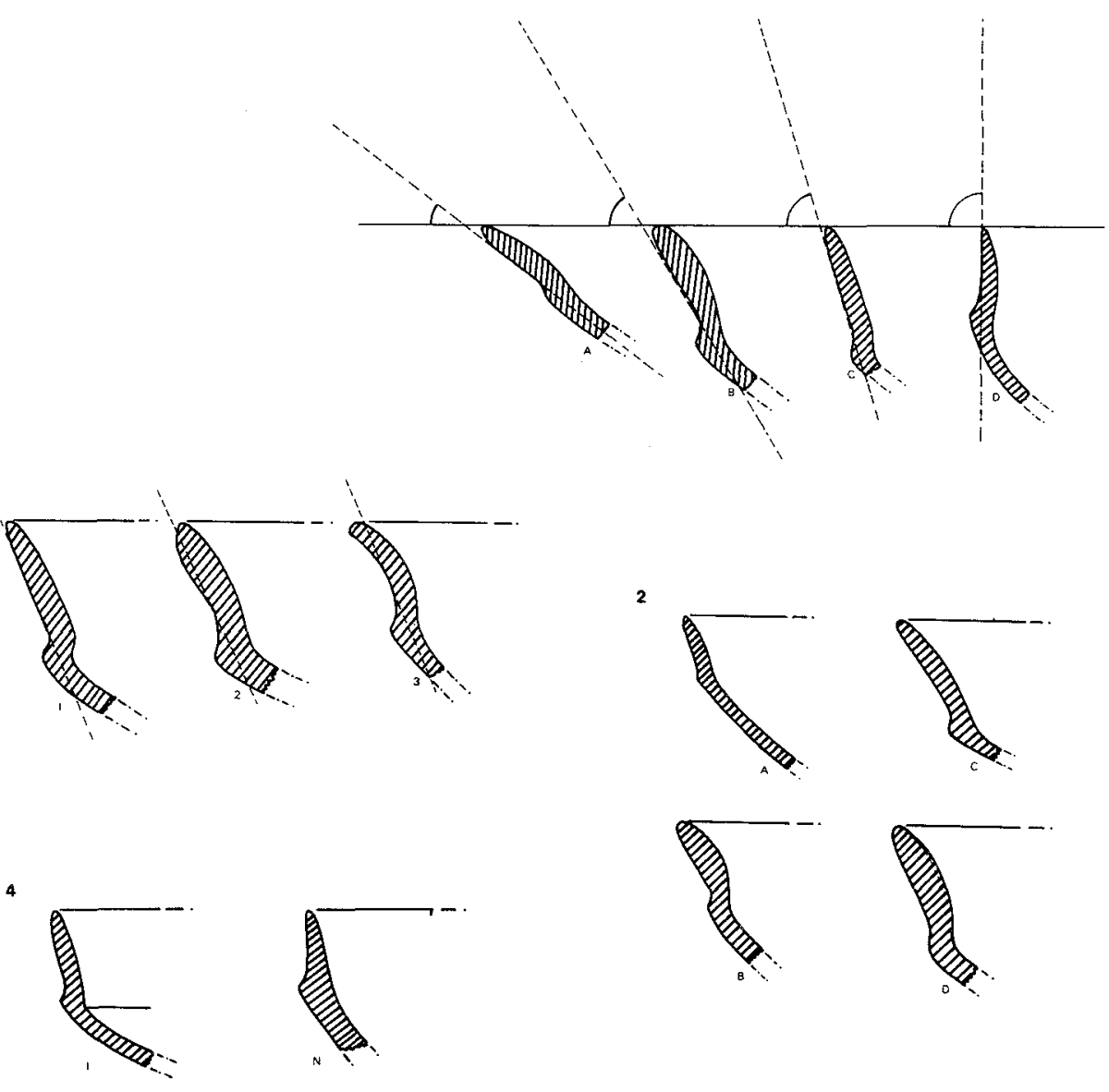

Fig. 1 Gráfico indicativo de los criterios utilizados para la delimitación de los «factores secundarios». 1, inclinación del borde y modo de medirla; 2 , relación longitud/grosor del borde; 3 , delineación del borde; 4 , inflexión interna. 


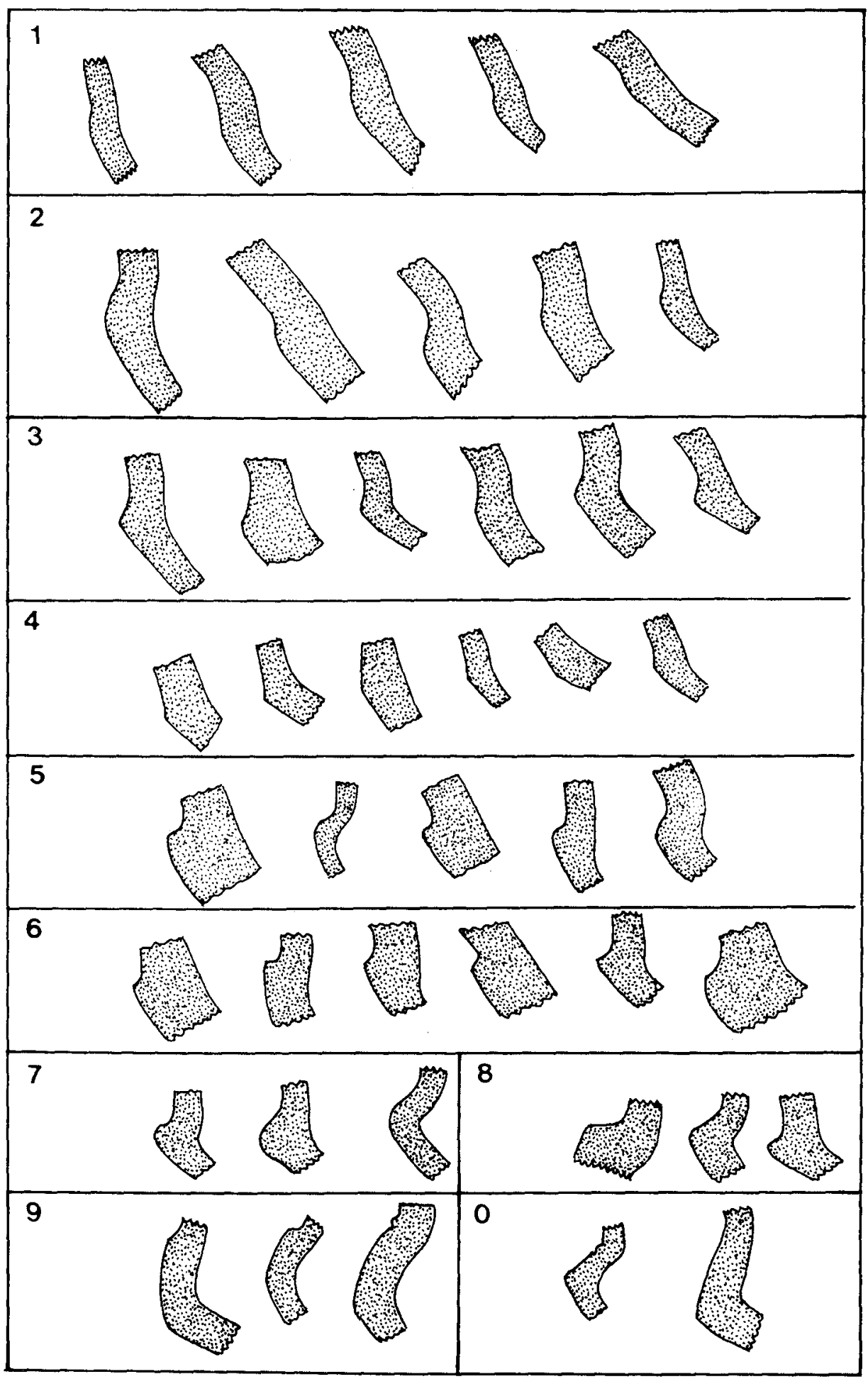

Fig. 2 Gráfico indicativo de los criterios utilizados para la delimitación de los tipos de carena, en el análisis de «factores secundarios». 
riante no se ha podido obtener una idéntica equidistancia, ya que en su determinación ha intervenido la sensación visual aplicada a los respectivos campos de inclinación. De esta forma nos hallamos ante las diferencias de amplitud siguientes: $\mathrm{A}=29^{\circ}, \mathrm{B}=27^{\circ}$, $\mathrm{C}=6^{\circ}, \mathrm{D}=18^{\circ}$ y E $=21^{\circ}$. Destaca el escaso margen del carácter $\mathrm{C}$ debido a que se trata en realidad de una variable intermedia entre los bordes abiertos y los verticales, como lo indica su denominación «de tendencia recta». Pero este campo lo consideramos significativamente necesario por cuanto semejante gradación se aparta de lo que aquí se entiende por «bordes abiertos», sin llegar a encajar entre los bordes rectos.

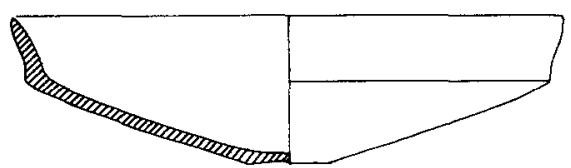

$\mathrm{B} 2 \mathrm{~b} 1 \mathrm{c} / \mathrm{B} 3 \mathrm{C} 1 \mathrm{I}$

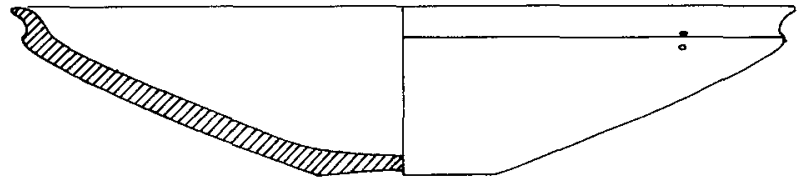

B1bla/A6A3I

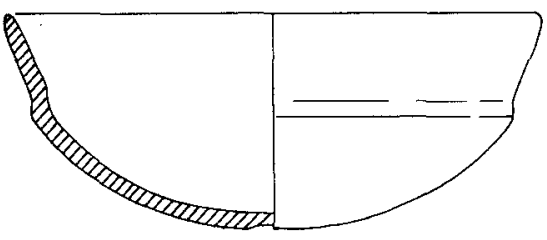

A2a2c/C1C1I

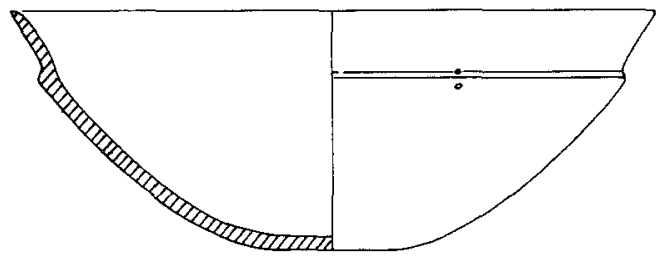

A 1a1a/ B6C1I

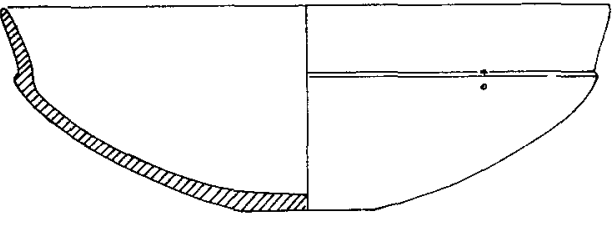

B1ala/ R6C1I

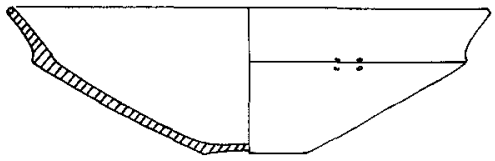

Albla/B3C1I

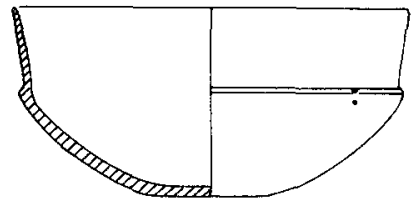

A2ala/C6ClI

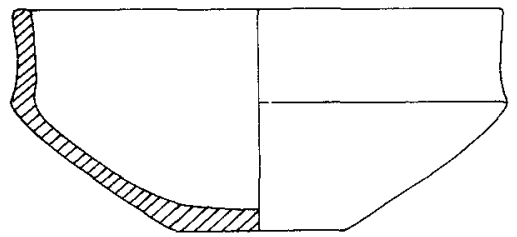

A $1 b 1 c / 03031$

Fig. 3 Gráfico ilustrativo de la denominación tipológica de las diversas variantes de las formas B7, definidas por factores primarios/factores secundarios. 
En el segundo grupo, las tres variables básicas - carenas simples, en hombrera y anchas - se han subdividido en otras secundarias con el fin de apurar al máximo el estudio analítico de este carácter formal. La separación entre los caracteres básicos está bien delimitada visualmente. En cambio, existen casi insensibles fronteras entre los caracteres secundarios. Para una mayor visualización de la gradación de la carena adjuntamos el pertinente gráfico que muestra una ordenación desde las carenas más simples e imperceptibles hasta las más acusadas y notorias.

Los restantes tres grupos no presentan estas dificultades por observar connotaciones más expresivas y objetivas, a pesar de lo cual se ilustran con otros gráficos.

La aplicación del sistema de variables o factores secundarios se ha realizado en dos fases. La primera, que es la que presentamos en este trabajo, tiende a una finalidad eminentemente cultural, buscando variables morfológicas características de la producción indistinta de estas vasijas en los dos horizontes en que aparecen (Peña Negra I y Peña Negra II). Para ello parece suficiente el número de ejemplares con que partimos, otorgando una caracterización de la producción alfarera del yacimiento con relación al resto de las estaciones contemporáneas del Sudeste.

Una segunda fase, con un objetivo básicamente cronológico requiere un volumen de muestras y un equitativo registro en ambos horizontes hoy por hoy inexistentes. Esta labor resta, pues, para el futuro de la investigación. Sólo entonces podremos tender a un análisis interno de cada período, con la correspondiente autopsia de cada uno de sus estratos integrantes.

\section{RESULTADOS}

El estudio estadístico que acompaña a estas líneas es obra de nuestro colaborador Miguel Ignacio Morales Moreno, quien tomó a sus espaldas la ingente tarea de realizar de forma manual los cómputos pertinentes al no poder pasarse el programa diseñado por él en el terminal del ordenador del Centro de Proceso de Datos del MEC situado en esta Universidad. A él se deben, igualmente, los gráficos que recogen estos resultados estadísticos.

Así las cosas, las 217 muestras procedentes de las excavaciones realizadas hasta 1981 que han sido sometidas al análisis de factores secundarios arrojan un total de 117 variantes reales.

Su ordenación en frecuencia, recogida en la tabla 2, se resume en la siguiente lista:

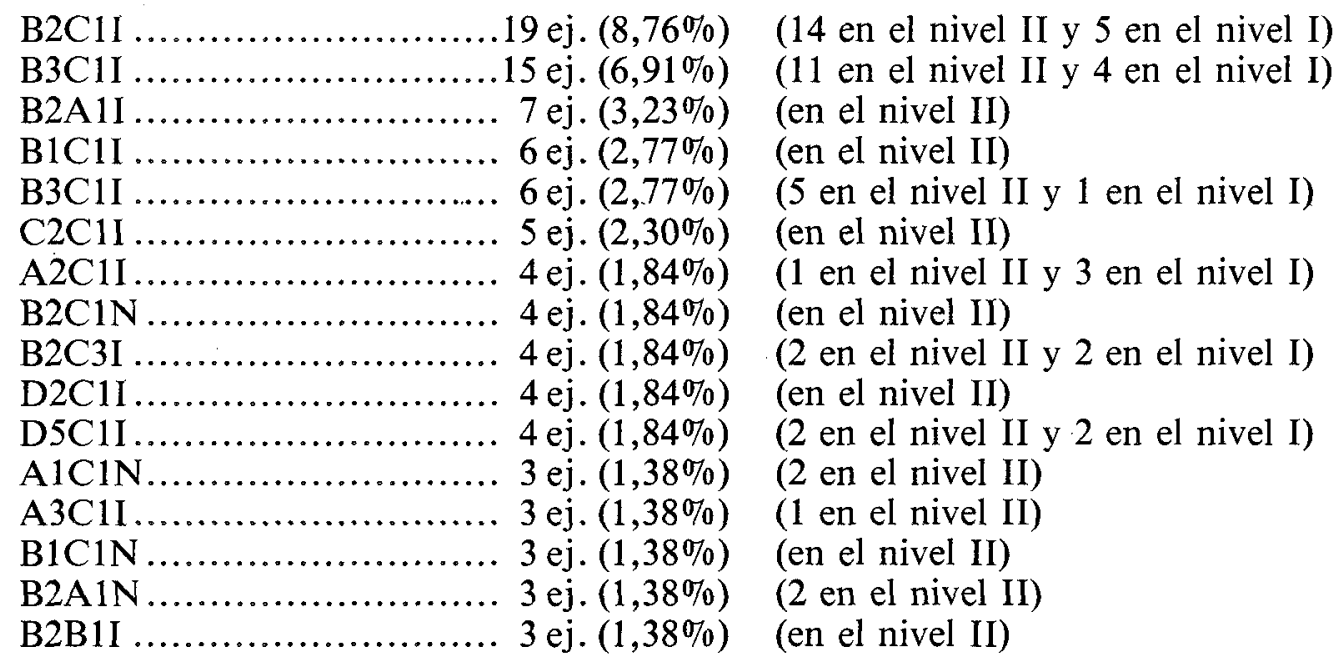




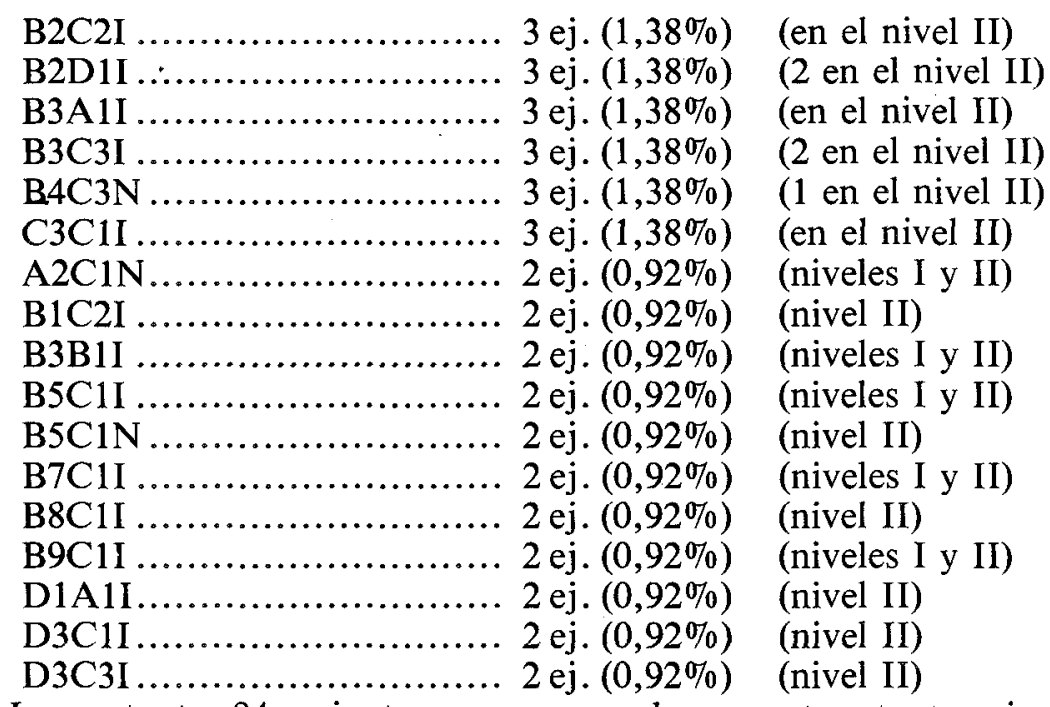

Las restantes 84 variantes se corresponden con otros tantos ejemplares, significando un porcentaje de 0,46 .

Las variantes predominantes B2C1I y B3C1I reúnen en lugar destacado a las cazuelas/cuencos de bordes abiertos, largos y delgados, con carenas simples redondeadas o acusadas, que marcan en el interior del vaso una inflexión.

El mayor porcentaje que existe en la fase Peña Negra I viene directamente condicionado por el mayoritario volumen de ejemplares proporcionados por los estratos correspondientes a este horizonte. Pero ello no es sino la exponencia de la amplia gama de variabilidad tipológica que afecta a dicha producción cerámica, que se verá sensiblemente reducida en el paso al horizonte Peña Negra II, al constituir una vajilla secundaria. Son muy escasos los ejemplares como para extraer siquiera conclusiones preliminares. Con todo se puede observar cómodamente en la precedente lista qué variantes parecen excusivas del Bronce Final y cuáles perduran en la etapa subsiguiente.

De la observación de estos primeros gráficos se desprende un fenómeno que parece tener, por su reiterada presencia, un significado cultural y cronológico. Afecta a las cazuelas con bordes rectos o verticales, que se decantan decididamente hacia el nivel inferior con 30 ejemplares (cifrados en 25 variantes), frente a 3 especímenes aparecidos en el período orientalizante, en forma de las variantes D2A1I y D5C1I.

Para un análisis más puntualizado se han tratado estos vasos cerámicos refiriéndolos a su área de procedencia del yacimiento. Este proceder permite comprobar si los resultados obtenidos del cómputo global del yacimiento son significativos, por ser representantes del mismo con la misma intensidad en todas sus áreas, o, por el contrario, su relevancia se encuentra en función de un determinado Sector. Si esto último ocurriera sería preciso cuestionarnos la causa de semejante fenómeno espacial, que podría tener una directa implicación temporal.

De los sectores en que se han llevado a cabo excavaciones sólo el IA (1976) y el II (1979) proporcionan un muestreo con fiabilidad suficiente al disponer de 48 y 146 ejemplares respectivamente. La escasa o nula representatividad de la forma B7 en los restantes sectores obliga a basar los resultados sobre las áreas precitadas, aunque en los gráficos se adjuntan los resultados de las muestras de los sectores IB y VII.

Para este análisis por sectores M. I. Morales ha diseñado tres tipos de gráficos. El primero (tablas 3 y 4 ) analiza por partes el valor de cada variable (sigla) refiriéndola en frecuencia relativa al Sector y su significación relativa a la totalidad de las muestras analizadas del yacimiento. Este gráfico incluye un cómputo suplementario realizado por niveles. 


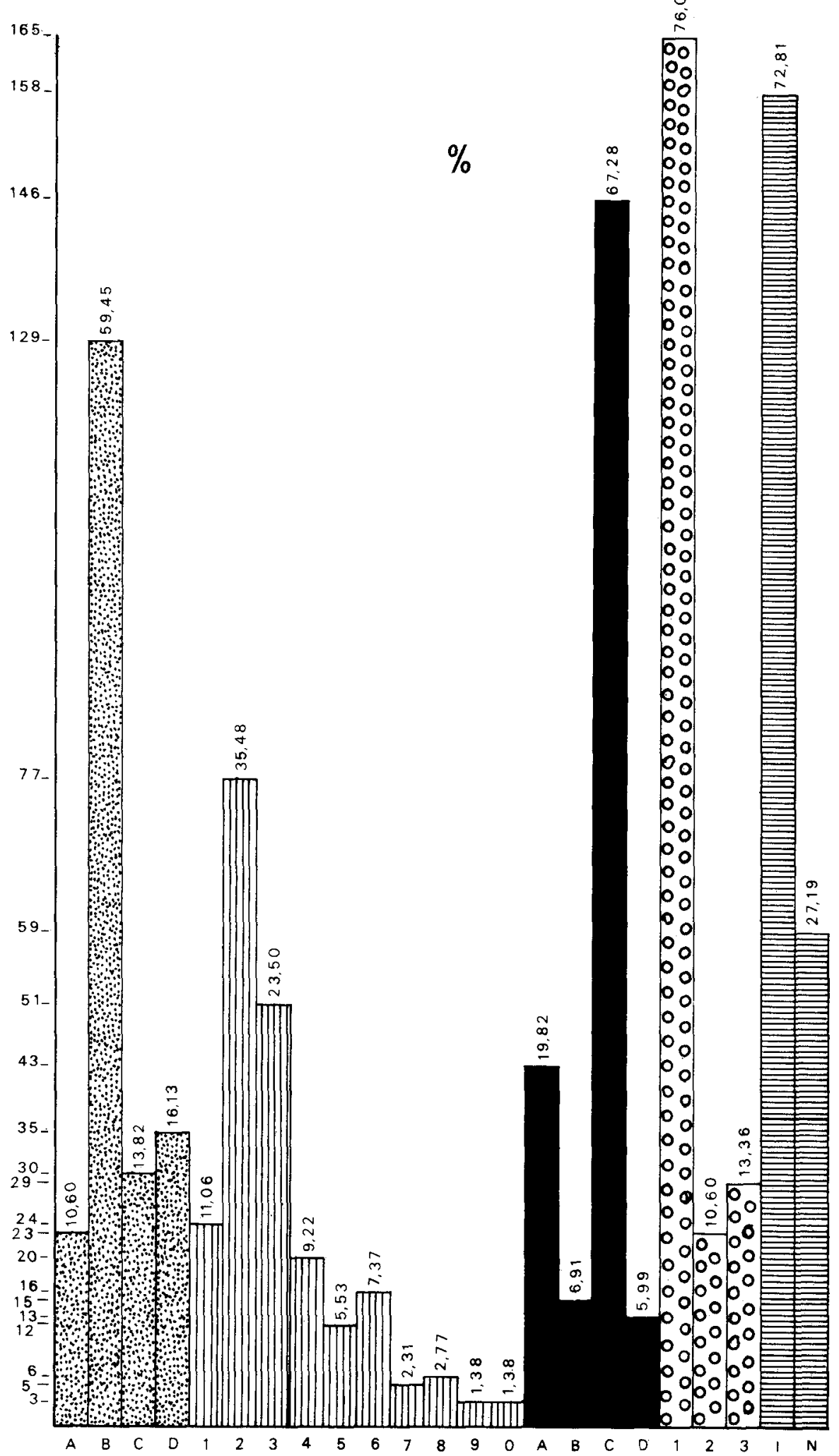

Fig. 4 Gráfico de frecuencia de las distintas variables de los grupos de factores secundarios. 

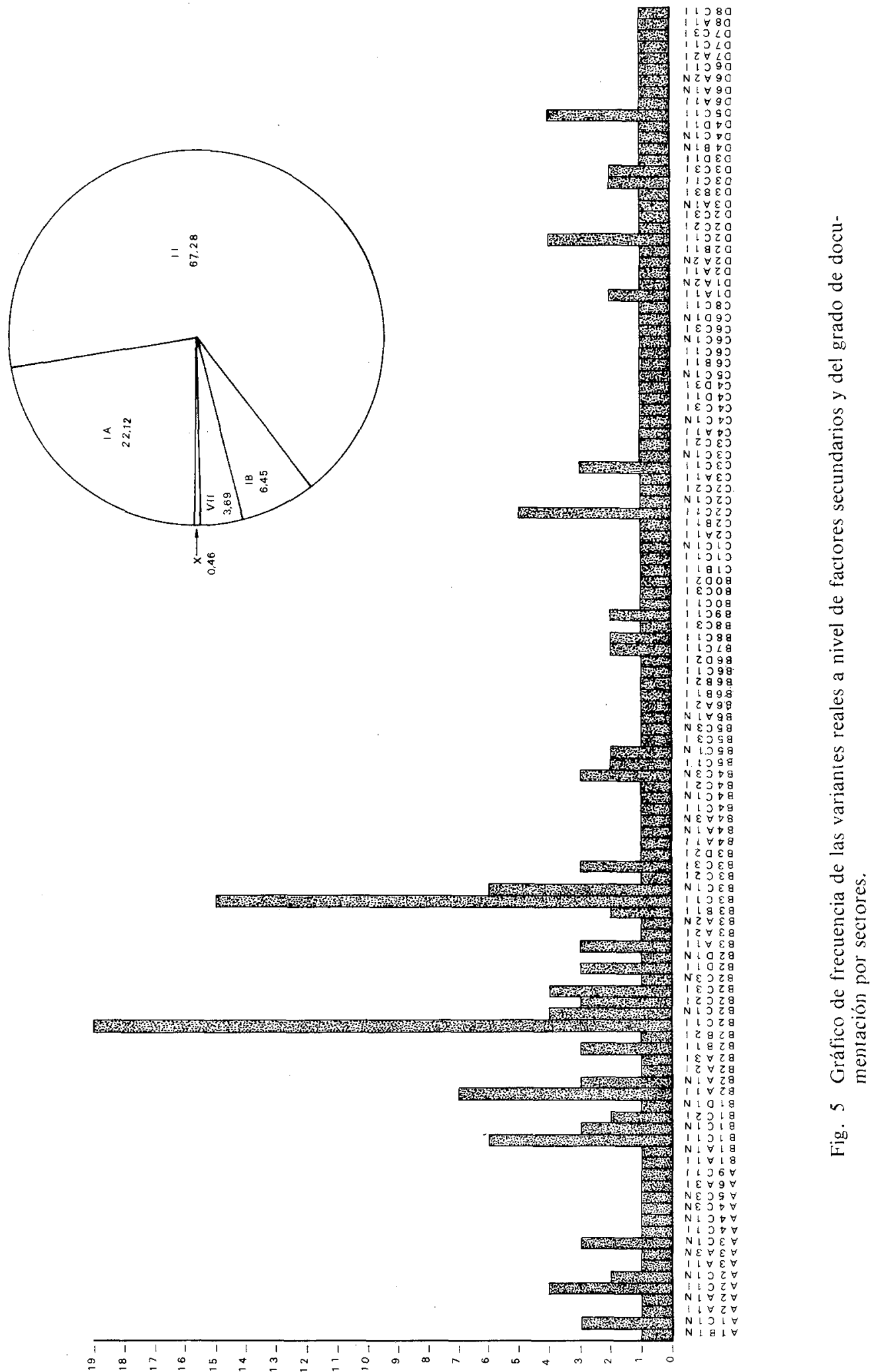
Un segundo gráfico (tablas 3 y 4) muestra las variables ya interconectadas en forma de variantes tipológicas, así como su frecuencia con relación al sector y a la totalidad y sus valores por niveles. Un porcentaje total $(100 \%)$ en la frecuencia relativa al Total implica la presencia de la variante en cuestión de forma exclusiva en ese sector del yacimiento.

El tercer gráfico (tablas 5 y 6 ) nos presenta en valores absolutos la frecuencia de las combinaciones de las variables entre los cinco grupos de significación establecidos.

\section{Sector IA (1)}

El primer grupo de variables cuenta con una frecuencia del $50 \%$ por parte del carácter $\mathrm{B}$, mientras ello se traduce en un $18,60 \%$ al referirlo al total. Este carácter se distribuye en valores sensiblemente iguales en ambos niveles (nivel I: 54,17; nivel II: $45,83)$.

Va seguido por el carácter A con un porcentaje del 27,08\% en el Sector y una frecuencia relativa a la totalidad del $56,52 \%$, que indica el elevado número de ejemplares con variable A hallados en el área IA. Este carácter aparece predominando ligeramente $(61,54 \%)$ en el nivel orientalizante, mientras en el propio del Bronce Final representa un $38,46 \%$.

El carácter $\mathrm{D}$, con un $12,5 \%$ respecto al setor y 17,14 respecto al total, se discrimina claramente hacia el nivel inferior.

En el segundo grupo de variables que contemplan el modo de carenación, predomina el carácter $2(31,25 \%)$ ligeramente sobre el $3(25 \%)$, y su aparición en el nivel I duplica el valor alcanzado en el nivel II. En el tercer grupo se destaca la presencia de los bordes largos y delgados (C) con un predominio del orden del $75 \%$, que se reparten casi por igual en ambos niveles.

El cuarto grupo viene representado mayoritariamente $(83,33 \%)$ por los bordes rectilíneos (1).

El último grupo resulta más aleatorio, ofreciendo sus caracteres constitutivos valores muy parecidos en ambos horizontes.

Las variables predominantes son $\mathrm{B} 2 \mathrm{C} 1 \mathrm{I}$ y $\mathrm{B} 3 \mathrm{C} 1 \mathrm{I}$ con una frecuencia de $8,33 \%$ respecto al Sector IA y de $21,05 \%$ y $26,67 \%$ respectivamente relativo al total de estas variantes.

\section{Sector II (2)}

En el primer grupo predomina considerablemente el carácter B, con un $60,27 \%$ que se traduce en un $68,22 \%$ relativo a la totalidad, indicándonos el elevado número de ejemplares procedentes de este Sector.

En su distribución por horizontes ofrece una clara concentración en el Bronce Final $(95,45 \%)$, representando únicamente un $4,55 \%$ en la etapa orientalizante.

En orden de frecuencia, viene seguido por el carácter D con un $18,49 \%$ en el Sector y un $77,14 \%$ en comparación con los restantes ejemplares hallados en el yacimiento. Resulta significativo que el $\mathbf{9 2 , 5 9 \%}$ se encuentre en el nivel II, contando el nivel superior sólo con un $7,41 \%$.

(1) Los fragmentos analizados se hallan publicados en GONZALEZ, 1979.

(2) La memoria de 1979, en que se da rendida cuenta de los ejemplares utilizados en este estudio, permanece todavía inédita en espera de su publicación por el Museo Arqueológico Provincial de Alicante, tras la pertinente autorización de la Subdirección General de Arqueología. 
Por lo que respecta a las carenas, es decir al segundo grupo de variables, existe nuevamente una preponderancia del carácter 2 con un $37,67 \%$ que significa con respecto al total un $71,43 \%$. Se distribuye con un 94,55 en el nivel inferior y con un 5,45 en el superior.

La carena simple acusada (3) le sigue en frecuencia, con un $20,55 \%$ en el Sector y un 58,82 referido al total. La concentración se efectúa en el nivel II $(93,33 \%)$.

En el tercer grupo, el labio largo y delgado (C) representa el $62,33 \%$ e idéntico porcentaje respecto al total. Como ocurre en todos los grupos, dado el mayor volumen de muestras procedentes de los estratos inferiores, se discrimina hacia el nivel II con un $92,31 \%$.

Los bordes cortos y delgados le siguen a continuación (A) con un $23,29 \%$ en el Sector y un $79,07 \%$ referido al total. La distribución por niveles sigue la línea de las restantes variables: $94,12 \%$ en el nivel inferior y 5,88 en el superior.

Además existe un neto predominio de los bordes rectilíneos con un $73,29 \%$ en el Sector y un $64,85 \%$ relativo al total. Con un valor de $92,52 \%$ se intengran en el nivel inferior.

En el quinto grupo los fragmentos que presentan inflexión en el interior constituyen un $76,03 \%$, concentrándose en el nivel II con el 93,69\%.

La absoluta discriminación que existe en el Sector II a favor del nivel inferior está condicionada por representar éste más de la mitad de las muestras analizadas en total.

Las variantes con más relevancia son B2C1I ( $7,53 \%$ relativo al Sector y $57,89 \%$ al total), B3C1I $(5,48 \%$ al Sector y $53,33 \%$ al total $)$, y B1C1I $(4,11 \%$ al Sector y sin presencia en el resto de los sectores excavados).

\section{Sector IB (3)}

Debido al escaso número de muestras halladas en este Sector, la significatividad de las frecuencias es mínima. No obstante, la tendencia general tipológica parece ser la misma.

En el primer grupo destacan los bordes abiertos (B) con el 64,29\%, significando únicamente el $6,98 \%$ respecto al total.

En el grupo de las carenas, el bajo número de las muestras señala frecuencias poco expresivas. Predominan las carenas simples redondeadas (2) y acusadas (3) con valores idénticos.

En el tercer grupo sólo aparecen los bordes largos y delgados, predominando en el nivel II.

Los bordes son rectilíneos (1) preferentemente. Y la mayoría de los ejemplares muestran inflexión interna $(85,71 \%)$.

La variante B2C1I se repite más veces $(21,43 \%)$, seguida con un valor de $14,29 \%$ por las variantes $\mathrm{B} 3 \mathrm{C} 1 \mathrm{I}$ y $\mathrm{C} 3 \mathrm{C} 1 \mathrm{I}$.

\section{Sector VII (4)}

Sólo a modo indicativo señalamos resultados de este sector. Predomina la inclinación B $(87,50 \%)$, las carenas simples acusadas (3), los bordes largos y delgados, rectilíneos y con inflexión interna.

(3) La memoria de 1978 se halla en prensa en la Serie de Trabajos Varios del SIP de la Excma. Diputación Provincial de Valencia y estamos a la espera de su publicación, tras haber entregado el original en 1979.

(4) Los escasos ejemplares recogidos en las campañas de 1980-81 en el Sector VII se publican en la memoria correspondiente, de inminente aparición en el vol. 17 del Noticiario Arqueológico Hispánico. 


\section{CONCLUSIONES GENERALES}

De una manera global, reuniendo y reagrupando ciertas variables del segundo grupo, se observa que existe una concentración de las carenas en hombrera en el nivel inferior, con sólo alguna anómala aparición en el nivel superior.

En su asociación con las variables del primer grupo se nos revela nuevamente este fenómeno. Con el carácter $\mathrm{A}$ aparece en dos ocasiones en ambos niveles. Con el $\mathrm{B}$, en 15 casos pertenecientes al nivel inferior, mientras sólo dos en el superior.

Con el carácter $C$ se asocia 7 veces y exclusivamente en el nivel II, de la misma forma que ocurre con las muestras de bordes rectos (D) combinadas aquí diez veces.

Es decir, que tanto la variable genérica de «carena en hombrera» como los «bordes de tendencia recta y rectos» son más fieles representantes de los estratos del Bronce Final.

Mientras que las carenas simples asociadas a bordes tumbados y abiertos aparecen a lo largo de toda la duración de la producción alfarera a mano, cubriendo ambos horizontes culturales de Peña Negra.

\section{LAS FORMAS B7 EN EL MARCO DE LA PROTOHISTORIA PENINSULAR}

En nuestro intento de dilucidar las tradiciones culturales que se depositan en el seno de las realizaciones materiales de Peña Negra I se observan diversas variantes carenadas integradas en bloque en nuestro tipo B7.

Por un lado, los pequeños cuencos de carena media pueden representar un enlace con los vasitos de la misma forma existentes en las fases finales del Bronce Valenciano, como los que proceden de Les Planetes de Benassal (GONZALEZ PRATS, 1978, 229, núm. 33) o en el Tabaiá de Aspe (NAVARRO MEDEROS, 1982, fig. 23, c). Molina los considera característicos de su Fase I del Bronce Final del Sudeste (MOLINA GONZALEZ, 1977, 15, núms. 15 y 17), aunque se encuentran igualmente en el yacimiento turolense de la Muela Pequeña del Rajo (ATRIAN JORDAN, 1957-58, fig. 6, 2).

Por otro lado, las formas con carena alta contarían con lejanos precedentes en el calcolítico portugués (LEISNER, 1965, lám. 2, 56; SCHUBART, 1973, fig. $10 \mathrm{n}$ ), aunque para el ejemplar de Barro, en Torres Vedras, se ha supuesto una cronología más tardía, desconectada del tholos (SCHUBART, 1971, 174). Este investigador germano señalaba en su ya célebre estudio sobre la cerámica bruñida del Bronce Tardío en el Sur y Oeste peninsular la posibilidad de que algunas formas carenadas se inspiraran en la tradición de las cazuenas campaniformes (ibídem, 173).

Esta derivación nos resulta evidente para nuestros ejemplares que ofrecen anchas hombreras de gradación 9 y 0 sobre las que en ocasiones se desarrolla una esmerada decoración incisa a base de reticulados o triángulos rellenos de trazos paralelos oblicuos y líneas de zig-zag que tanto deben a la tradición campaniforme.

Las variantes B7A que adoptan carenas simples sin hombrera (gradación 1 a 4) nos parecen derivadas o. inspiradas en los cuencos carenados que a partir del Bronce Medio tienden a situar la línea de inflexión en el tercio superior del vaso, tal y como se encuentran yả formados en el horizonte de Cogotas I e incluso en la posible etapa precedente de este complejo meseteño que algunos autores han entrevisto en el yacimiento vallisoletano de La Plaza (DELIBES-FERNANDEZ, 1981, 63 ss.). El origen de estas formas con carena alta simple se aprecia tanto en la Cueva de Arevalillo en Cega en un momento en que «el boquique y los temas incisos de diseño simple parecen haber ganado terreno al campaniforme» (FERNANDEZ POSSE, 1981, 74), como en la Cueva de la Vaquera de Torreiglesias, asociadas con cerámicas de tradición campaniforme y datadas 
hacia el siglo XIV a. de J.C. (ZAMORA CANELLADA, 1976, fig. XI, 141), o en el Cerro del Ecce Homo cuya primera fase, correspondiente al horizonte de Cogotas I, ha sido fechada en el siglo XII a. de J. C. (ALMAGRO-FERNANDEZ, 1980, figś. 8 ss.).

Otra característica de estas formas de carena alta es la de ofrecer un cuerpo troncocónico (B7A1b), detalle que nos acerca a los tipos meseteños aquellos ejemplares semejantes del Sudeste. Asimismo, aunque en los numerosos yacimientos de las Cogotas Antiguas se dan bordes abiertos y otros verticales y ligeramente reentrantes, parece existir una predilección por estos últimos, preferencia que decrece en el Bronce Final en que prevalecen formas con bordes abiertos.

El hecho de que en Peña Negra I tengan entidad los bordes rectos o verticales (D) de la que no disfrutan los estratos orientalizantes, nos llegaría a indicar quizá esta influencia de las formas de la Meseta.

Por su parte, las variantes que ostentan carenas en hombrera más o menos acusadas (gradación 5 a 8) tienen un área de distribución exclusivamente meridional, de una manera aproximada por debajo de la línea que une el Cabo de Peniche con el Cabo de la Nao. Los yacimientos lusos del Bronce Final presentan, al lado de formas con carenas simples, otras con hombrera en donde se sitúan pequeños engrosamientos con taladro vertical, característica que define todo el ámbito tartésico, entendido aquí sensu latu desde Ourique al Vinalopó (SPINDLER-DA VEIGA, 1973 y 1974). La presencia de la hombrera en estos ejemplares portugueses, algunos de los cuales alardean de su peculiar decoración bruñida externa, se debe con seguridad a las formas del Bronce II del SO, como los cuencos de tipo Santa Vitoria o del tipo Odivelas (SCHUBART, 1975), uno de los cuales estaba acompañado en Lapa do Suão por cazuelas de carena alta (SPINDLER-DA VEIGA, 1974, fig. 4).

Los cuencos y cazuelas de carena alta constituyen, entre otros, los hallazgos más característicos del área nuclear tartéssica y su origen todos los autores coinciden en situarlo en la tradición indígena (SCHUBART, 1971, 171: BELEN et Alii, 1977, 357; ARTEAGA, 1977; TEJERA GASPAR, 1978, 189; MOLINA GONZALEZ, 1978, 203). La comparación entre las formas de los dos focos principales tartésicos -el de Huelva y el Bajo Guadalquivir - llevó a varios investigadores a diferenciar el grupo onubense, con una mayor calidad de los vasos así como la presencia de carenas pronunciadas, del grupo hispalense cuyas formas son menos carenadas y de peor calidad (LOPEZ ROA, 1978, 150 ss.; MOLINA GONZALEZ, 1978, 219). El criterio de prioridad cronológica del grupo de Huelva (IX-VIII a. de J. C.) sobre el del Bajo Guadalquivir (VIII-VII a. de J. C.) no resulta válido para el grupo del Sudeste, en donde conviven - dentro de un alto grado de calidad de los vasos - las dos tendencias de las carenas, como puede observarse en el Cerro del Real, Peñón de la Reina o la propia Peña Negra.

Más allá de los límites geográfico-culturales señalados, estas formas se enrarecen, indicándonos el alejamiento de las áreas septentrionales de la zona de origen y desarrollo de este específico tipo de vasija bruñida. Con todo, y ello de forma excepcional, podrían asimilarse a la línea tipológica genérica el ejemplar con hombrera corta y borde vertical de la Muela Pequeña del Rajo (ATRIAN JORDAN, 1957-58, fig. 5, 5) y los procedentes de las tumbas 138, 164 Y 175 de la necrópolis de Agullana (PALOL, 1958, figs. 124, 146 y 157), así como algunos cuencos del Bronce Final del Mediodía francés (LOUIS-TAFFANEL, 1958, fig. 10; MERLIN, 1974, 48, fig. 4, 2), cuyos prototipos algunos autores rastrean en las formas de los túmulos del Bronce Medio de Haguenau (SCHAEFFER, 1926, figs. 34 s, 36 b, 38 p, 39 k, 47 c, 54 d y 60 h).

Sin embargo, un área en donde estas formas con carena alta alcanzan nuevamente cierta entidad y cuyo cotejo podría traernos en el futuro alguna sorpresa, es el mundo itálico. Allí aparecen en ambientes subapenínicos y previllanovianos, asociadas con otros vasos con asas de apéndice de botón (LOLLINI, 1979, fig. 1, 18 y 19). Resulta al- 
tamente revelador el hecho de que los apéndices o protuberancias presentes en los labios de las vasijas bruñidas de Saladares y Peña Negra (ARTEAGA-SERNA, 1975, lám. VI, 40) precisamente se documenten en los ambientes itálicos: en Cortine di Sta. María in Campo (LOLLINI, 1979, fig. 1, 22), datado en los siglos XII-X a. de J. C.; en Porto Perone, Leporano, en un ambiente tardoapenínico con cerámicas bruñidas carenadas y asas «cornutas» y fondos de cabaña (LO PORTO, 1963, fig. 20), o en Rocca di Roffeno (KRUTA, 1974, fig. 10, 25). En el Trentino Alto, diversos vasos que se decoran con motivos geométricos desarrollan pequeños apéndices triangulares en sus bordes (FUGAZZOLA, 1974, fig. 9). Semejante detalle resulta, a mayor abundamiento, habitual en la cultura villanoviana, como se puede observar en cuencos y tapaderas de urnas de la necrópolis del Caselle di Villanova o en la de Ca dell’Orbo (VITALI et Alii, 1979, lám. II, 5 y figs. 15-23).

Estas diversas valoraciones realizadas sobre las principales variantes del tipo B7 de Peña Negra I permiten entrever las distintas líneas de inspiración e influencia formal que, en nuestra opinión, concurren en dichas formas cerámicas, situándolas en el contexto de tipos similares desarrollados en suelo peninsular desde finales del Bronce Medio y durante el Bronce Final. El área geográfica que cubren estas vasijas se ciñe exclusivamente al territorio que asiste al desenvolvimiento del mundo tartéssico geométrico. En este sentido, la vocación meridional de Peña Negra queda bien patente. Pero la presencia de una técnica peculiar y característica del Sudeste como es la decoración incisa, que utiliza un repertorio de motivos geométricos de tradición campaniforme, y la entidad de las formas con carena simple y borde vertical, nos induce a contemplar la posibilidad de que en el espectro material del primer horizonte de la Peña Negra debamos dar cabida a una influencia de componentes culturales evidentes que emanan de las tradiciones formales e incluso decorativas del postcampaniforme y de la cultura de Cogotas I, y que cubren en las mesetas todo el II milenio precristiano. La existencia de vasijas carenadas con hombrera ancha similares en forma y casi en decoración a las cazuelas campaniformes por un lado, y por otro de vasos con carenas altas de tipo simple similares a las que en Cogotas I se decoran con motivos incisos, excisos o con la técnica de boquique, hablarían en este sentido. Consideramos sumamente ilustrativo comparar estas cazuelas con decoración incisa, de la que es fiel exponente el ejemplar recién publicado de Los Saladares (ARTEAGA-SERNA, 1979-80, fig. 22), con sus predecesoras del mundo campaniforme para concluir en una derivación que aún hoy, debido a la escasez de documentos intermedios (5), no acaba de estar confirmada, pero que ya en 1971 fue propugnada por el profesor Schubart.

Evidentemente, la sofisticada decoración que ostentan los bordes de estas cazuelas, en donde interviene la concepción de metopas y el realce otorgado por el empleo de la pintura, es signo evidente de que la vieja tradición se ha permeabilizado con las nuevas modas decorativas al uso, en donde adquiere un lugar relevante la decoración de zonas rellenas de pintura roja entre aquéllas que tienen incisión, y que, en definitiva, no era sino una técnica más de conseguir un bicromismo al hallarse los motivos incisos con incrustación de pasta blanca.

Ahora bien, Ios sorprendentes hallazgos efectuados en la Mesa de Setefilla por la doctora Aubet, documentándose una facies de inicios del Bronce Medio que viene a cubrir el vacío existente en esta época en Andalucía occidental, parecen significar el origen de algunos elementos que van a caracterizar el Bronce Final meridional (soportes-

(5) Es preciso traer a colación aquí las vasijas de Cogotas I del Cerro del Ecce Homo, por cuanto en varias de ellas se documenta una decoración incisa de tradición campaniforme (ALMAGROFERNANDEZ, 1980, fig. 32). 
carrete, decoración bruñida (6), decoración pintada) y entre los que se encuentran unos cuencos bruñidos de carena media y alta. Si se prodigan en el futuro estos hallazgos, obteniendo un mayor repertorio tipológico, dispondríamos en el II milenio de Andalucía de antecedentes de ciertas formas de cuencos y cazuelas del Bronce Final. Habría que señalar, no obstante, la similitud que existe entre los cuencos de carena alta simple cuyos labios se decoran con zig-zag incisos (AUBET SEMMLER, 1981, figs. 2, f y 4, d) y algunos de la fase Cogotas I de la Cuesta del Negro, datada hacia el siglo XII a.de J. C. (MOLINA-PAREJA, 1975, núms. 77 y 131 entre otros).

La tradición formal y decorativa campaniforme-Cogotas I se encontraría a la par con otra inspiración meridional y del SO a la que es preciso hacer responsable de la teñdencia de generar carenas con hombreras, si es que en un futuro no se llega a demostrar que tanto unas como otras son una misma evolución de las cazuelas campaniformes. El análisis aplicado a las muestras de Peña Negra nos caracteriza, además, un aspecto de la producción alfarera del yacimiento, diferenciándose del desarrollo que existe en Los Saladares, en donde se propone una posible gradación cronológica en la aparición de las fuentes de carena alta y bordes largos y delgados (fase IA1) y de los cuencos de carena alta con bordes cortos y gruesos (fase IA2) (ARTEAGA-SERNA, 1979-80, 105). Este fenómeno es una muestra más de la variedad y riqueza de personalismos locales dentro de un mismo fondo cultural, realidad que cada día se nos manifiesta mejor en el estudio de las comunidades peninsulares del Bronce Final.

Alicante, febrero de 1983

\section{BIBLIOGRAFIA}

ALMAGRO GORBEA, M. y FERNANDEZ GALIANO, D., 1980. Excavaciones en el Cerro Ecce Homo (Alcalá de Henares, Madrid). Madrid.

ARTEAGA MATUTE, O., 1977. «Las cuestiones orientalizantes en el marco protohistórico peninsular». Cuadernos de Prehistoria de la Universidad de Granada, II, 301-320.

ARTEAGA, O. y SERNA, M. R., 1975. «Los Saladares-71». Noticiario Arqueológico Hispánico, Arqueología 3, 7-140.

- 1979-80. «Las primeras fases del poblado de Los Saladares (Orihuela, Alicante)». Ampurias, $41-42,65-137$.

ATRIAN JORDAN, P., 1957-58. «Sobre un yacimiento de la Primera Edad del Hierro en la provincia de Teruel». Ampurias, 19-20, 244-52.

AUBET SEMMLER, M. ${ }^{\text {a }}$ E., 1981. «Sepulturas de la Edad del Bronce en la Mesa de Setefilla (Sevilla). Madrider Mitteilungen, 22, 127-149.

BELEN, M., FERNANDEZ MIRANDA, M. y GARRIDO, J. P., 1977. Los orígenes de Huelva. Excavaciones en los Cabezos de San Pedro y La Esperanza. Huelva Arqueológica, III. Huelva.

DELIBES, G. y FERNANDEZ, J., 1981. «El castro protohistórico de la Plaza en Cogeces del Monte (Vallảdolid). Reflexiones sobre el origen de la fase Cogotas I». Boletín del Seminario de Estudios de Arte y Arqueología, XLVII, 51-70.

FERNANDEZ POSSE, M. D., 1981. «La Cueva de Arevalillo de Cega (Segovia)». Noticiario Arqueológico Hispánico, 12, 45-84.

(6) La cerámica con decoración bruñida del estrato XV de la Mesa de Setefilla significaría un eslabón entre esta técnica documentada en Valencia de la Concepción y la propia del Bronce Final tartéssico, que únicamente se había hallado en el último cuarto del Il milenio AC en el Ecce Homo (ALMAGROFERNANDEZ, 1980, 104, fig. 23). 
FUGAZZOLA DELPINO, M. A., 1974. «Ceramiche protostoriche dal Trentino Alto adige nel Tiroler Landesmuseum di Innsbruck». Rivista di Studi Preistoriche, XXIX, 1, 179-211.

GONZALEZ PRATS, A., 1977-78. «Primeros resultados de las excavaciones realizadas en el yacimiento protohistórico de la Peña Negra de Crevillente, Alicante». Pyrenae, 13-14, 121-135.

- 1978 «Excavaciones en el poblado de la Edad del Bronce de Les Planetes, Mas d'en Serrans, Benassal (Castellón)». Cuadernos de Prehistoria y Arqueología Castellonense, 5, 207-237.

- 1979 Excavaciones en el yacimiento protohistórico de La Peña Negra, Crevillente, Alicante $11 .{ }^{a}$ y 2. ${ }^{a}$ campañas). Excavaciones Arqueológicas en España, 99. Madrid.

KRUTA, L., 1974. «Un abitato preistorico nell'Appennino bolognese. Relazione preliminare». Rivista di Science Preistoriche, XXIX, 1. 437-451.

LEISNER, G. y V., 1965. Die megalithgräber der Iberischen Halbinsel. Der Western. Tafeln. Berlin.

LO PORTO, G. F., 1963. «Leporano (Taranto). La stazione protostorica di Porto Perona». Atti dell'Academia Nazionale dei Lincei, XVII, 280-380.

LOLLINI, D., 1979. «II Bronzo Finale nelle Marche (Ancona)». Rivista di Studi Preistoriche, XXXIV, $1-2$ $179-215$.

LOPEZ ROA, C., 1978. «Las cerámicas alisadas con decoración bruñida». Huelva Arqueológica, IV, 145180.

LOUIS, M., TAFFANEL, J. y O., 1958. Le Premier Age du Fer Languedocien, II. Les nécropoles à incineration. Bordighera-Montpellier.

MERLIN, G., 1974. "Grotte du Gouffre-Faille, gisement du Bronze Final (Alpes maritimes)». Bulletin de la Societé Prehistorique Française, 71 (CRSM, 2), 45-48.

MOLINA GONZALEZ, F., 1977. La cultura del Bronce Final en el Sudeste de la Península Ibérica. Resumen de Tesis Doctoral. Granada.

- 1978 «Definición y sistematización del Bronce Tardío y Final en el Sudeste de la Península Ibérica». Cuadernos de Prehistoria de la Universidad de Granada, 3, 159-232.

MOLINA, F. y PAREJA, E., 1975. Excavaciones en la Cuesta del Negro (Purullena, Granada). E. A. E., 66. Madrid.

NAVARRO MEDEROS, J. F., 1982. «Materiales para el estudio de la Edad del Bronce en el valle medio del Vinalopó (Alicante)». Lvcentvm, 1, 19-70.

PALOL SALELLAS, P., 1958. La necrópolis hallstáttica de Agullana (Gerona). Bibliotheca PraehistóriHispana, I. Madrid.

SCHAEFFER, F. A., 1926. Les tertres funeraires prehistoriques dans la Fôret de Haguenau, I. Les tumulus de l'Age du Bronze. Bruxelles.

SCHUBART, H., 1971. "Acerca de la cerámica del Bronce Tardío en el Sur y Oeste peninsular». Trabajos de Prehistoria, 28, 153-182.

- 1973 «Tumbas megalíticas con enterramientos secundarios de la Edad del Bronce en Colada de Monte Nuevo de Olivenza». XII Congreso Nacional de Arqueología, 175-190.

SPINDLER, K. y DA VEIGA, O., 1973. «Der Spätbronzezeitliche kuppelban von der Roça do Casal do Meio in Portugal». Madrider Mitteilungen, 14, 60-108.

- 1974 «Das vorgeschichtliche fundmaterial aus der Gruta do Carvalhal (Portugal)». Madrider Mitteilungen, 15, 28-76.

TEJERA GASPAR, A., 1978. «El Bronce Final del Bajo Guadalquivir y su problemática». Huelva Arqueológica, IV, 181-196.

VITALI, D. et Alii., 1979. La necropoli villanoviana di Ca dell'Orbo a Villanova di Castenaso. Problemi del popolamento dal IX al VI s. AC. Bologna.

ZAMORA CANELLADA, A., 1976. Excavaciones en la Cueva de la Vaquera, Torreiglesias (Segovia). Segovia. 

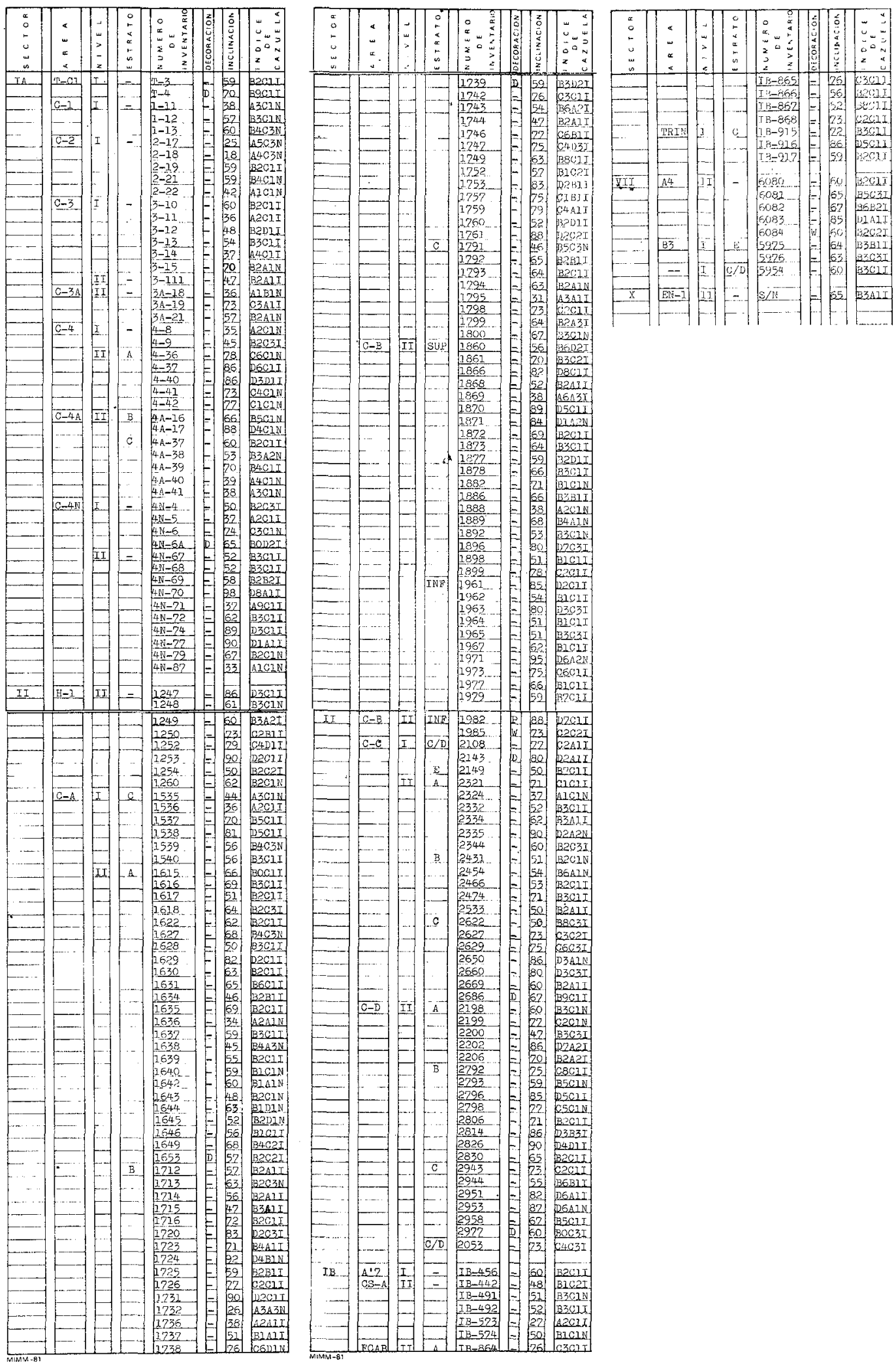

Tabla 1 Listado de las variantes formales por orden de inventario. 

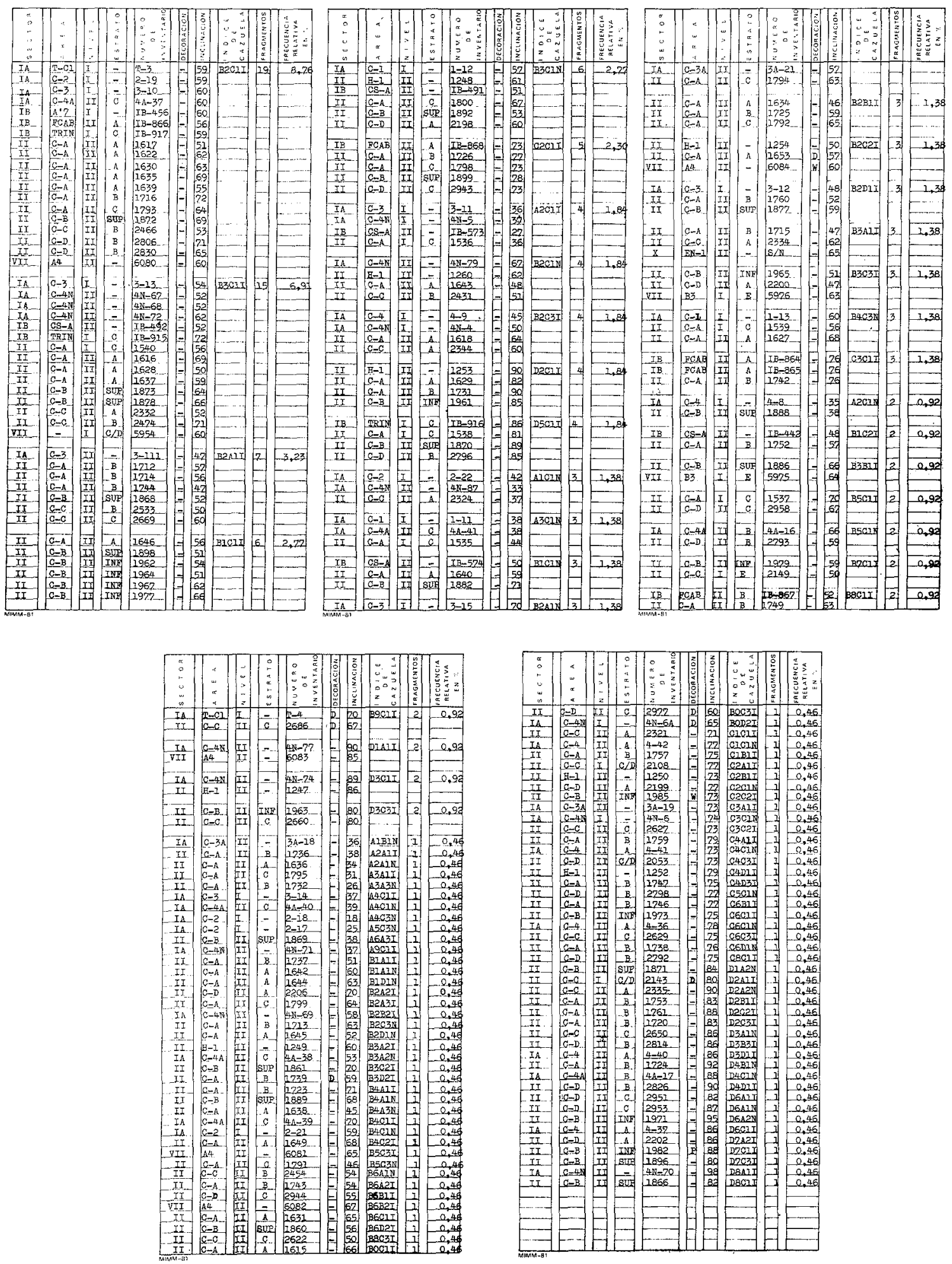

Tabla 2 Listado de los grupos de variantes por orden de frecuencia. 

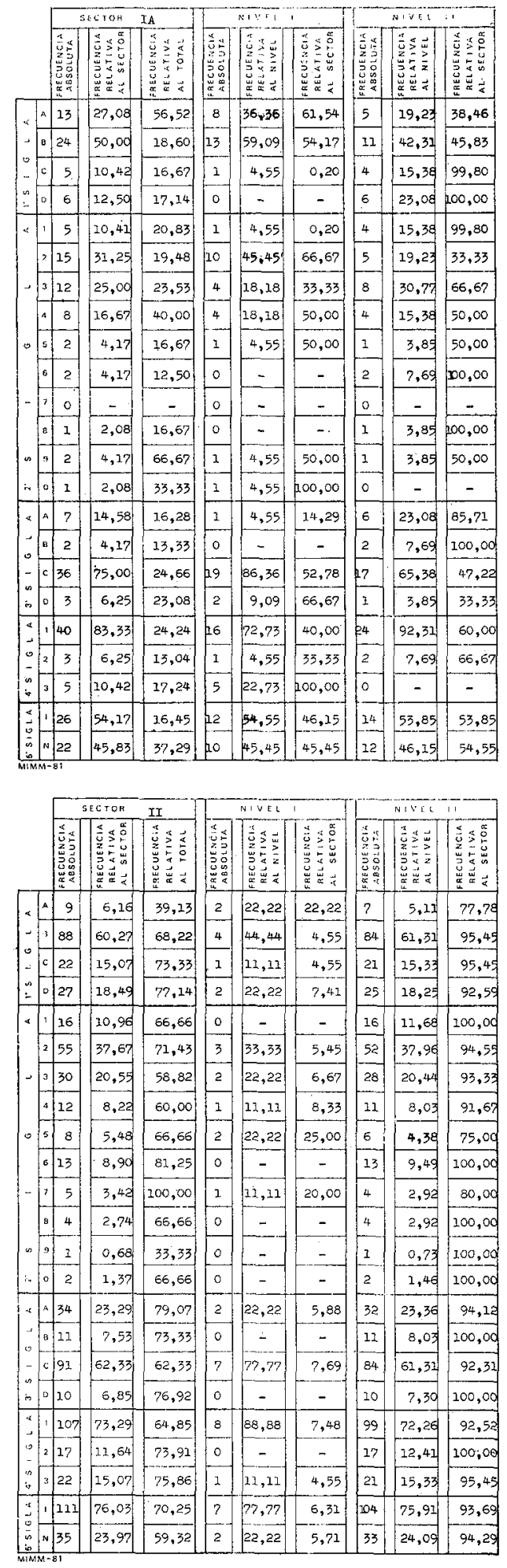
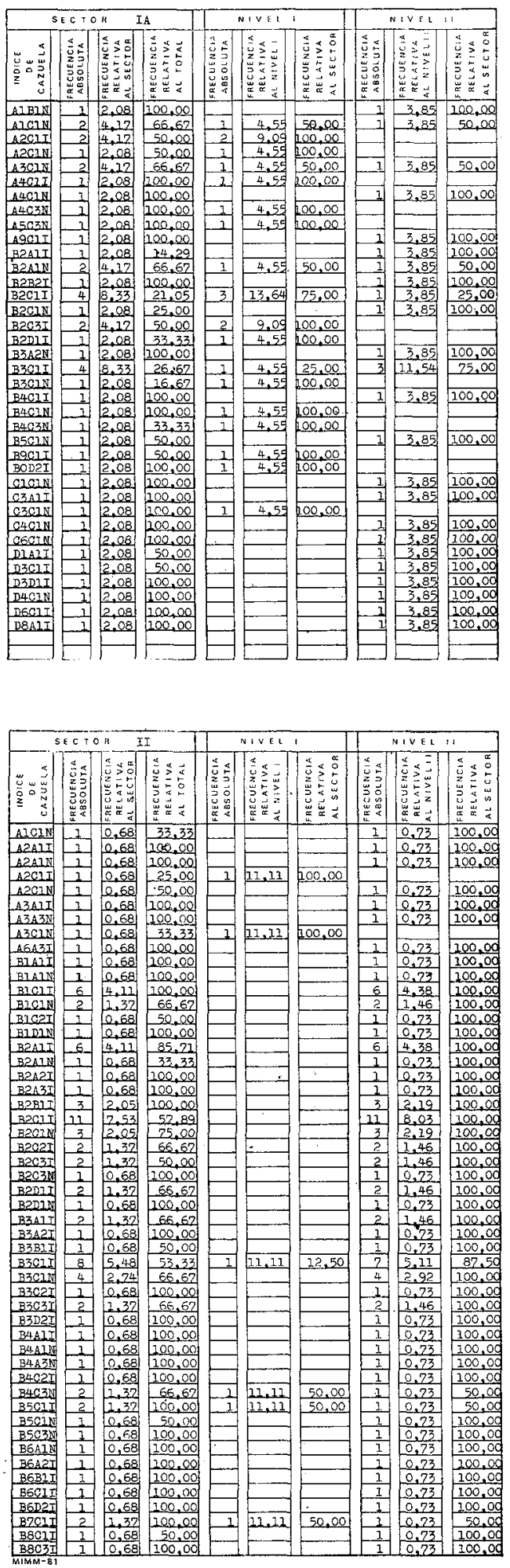

Tabla 3 Listado de frecuencia de variables y variantes por sectores y niveles. 


\begin{tabular}{|c|c|c|c|c|c|c|c|c|}
\hline 等 & 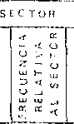 & 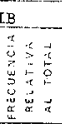 & $\begin{array}{l}0 \\
3 \\
3 \\
3 \\
0 \\
0\end{array}$ & 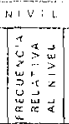 & 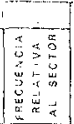 & 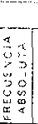 & $\mid$ & 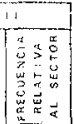 \\
\hline 1 & 7,14 & 4,35 & 0 & - & - & 1 & 10,00 & 100,00 \\
\hline 9 & 64,29, & 6,98 & 3 & 75,00 & 33,33 & 6 & 150,00 & 66,67 \\
\hline 3 & $21,43]$ & 10,00 & 0 & - & - & 3 & 30,00 & 100,00 \\
\hline 1 & 7,14 & 2,86 & 1 & 25,00 & 100,00 & 0 & - & - \\
\hline 2 & $\mathrm{I4}, 29$ & 8,33 & 0 & - & - & 2 & 20,00 & 100,00 \\
\hline 5 & 35,71 & 6,49 & 2 & 50,00 & 40,00 & 3 & 30,00 & 50,00 \\
\hline 5 & 35,71 & 9,80 & 1 & 25,00 & $20, \infty$ & 4 & 40,00 & 80,00 \\
\hline o & - & - & 10 & - & - & 0 & - & - \\
\hline I & $7,1.4$ & $.8,33$ & 1 & 25,00 & $100, \infty$ & 0 & - & - \\
\hline 0 & - & - & 0 & - & - & 0 & - & $=$ \\
\hline 0 & - & - & 0 & - & - & 0 & - & - \\
\hline$I$ & 7,14 & 26,65 & 0 & - & - & 1 & 10,00 & 100,00 \\
\hline 0 & - & - & 0 & - & - & 0 & - & - \\
\hline 0 & - & - & 0 & - & - & 0 & - & - \\
\hline 0 & - & - & 0 & - & - & 0 & - & - \\
\hline 0 & - & - & 0 & - & - & 0 & - & - \\
\hline 14 & 100,00 & 9,59 & 14 & 000,00 & 28,57 & 10 & 100,00 & 71,43 \\
\hline 0 & - & - & 10 & - & - & 0 & - & - \\
\hline 13 & 92,86 & 7,88 & 4. & 200,00 & 30,77 & .9 & 90,00 & 69,23 \\
\hline 1 & 7,24 & 4,35 & 0 & - & - & 2 & 10,00 & 100,00 \\
\hline 0 & - & - & 0 & - & - & 0 & - & - \\
\hline 12 & $85,7]$ & 7,59 & 4 & 100,00 & 33,33 & 8 & 80,00 & 66,67 \\
\hline 2 & 14,29 & 3,39 & 0 & - & -3 & 2 & 20,00 & 100,00 \\
\hline
\end{tabular}

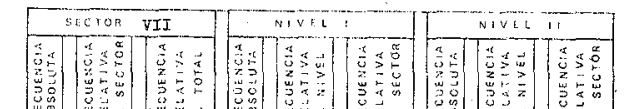

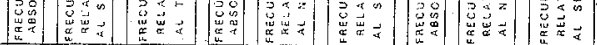

\begin{tabular}{|c|c|c|c|c|c|c|c|c|c|}
\hline & 0 & - & - & 0 & - & - & 0 & - & - \\
\hline & $?$ & $87,50 \mid$ & 5,43 & 3 & 100,00 & 42,86 & 4 & $80,00)$ & 57,24 \\
\hline & 0 & - & & 0 & - & - & 0 & - & - \\
\hline & 1 & $|12,50|$ & 2,86 & 0 & - & - & 1 & {$[20,00]$} & 100,00 \\
\hline & 1 & 12,50 & 4,17 & 0 & - & - & 1 & 20,00 & $100, \infty$ \\
\hline & 2 & 25,00 & 2,50 & 0 & - & - & 2 & 40,00 & 100,00 \\
\hline & 3 & 37,50 & 5,88 & 3 & 100,00 & 100,00 & 0 & - & - \\
\hline & o & - & - & 0 & - & - & 0 & - & - \\
\hline & I & 12,50 & 8,33 & 0 & - & - & 1 & 20,00 & 100,00 \\
\hline & 1 & 12,50 & 6,25 & 0 & - & - & 1 & 20,00 & 100,00 \\
\hline & 0 & - & - & $\circ$ & - & - & 0 & - & - \\
\hline & 0 & - & - & 0 & - & - & 0 & - & - \\
\hline & 0 & - & - & 0 & - & - & 0 & - & - \\
\hline & 0 & - & - & 0 & - & - & 0 & - & - \\
\hline & 1 & 22,50 & 2,33 & $\circ$ & - & - & 1 & 20,00 & 100,00 \\
\hline & 2 & 25,00 & 23,33 & 1 & 33,33 & 50,00 & 1 & $20, \infty$ & 50,00 \\
\hline & 5 & 62,50 & 3,42 & 2 & $(66,67)$ & 40,00 & 3 & 60,00 & 60,00 \\
\hline & 0 & - & - & $\circ$ & - & - & 0 & - & - \\
\hline & 4 & 50,00 & 2,42 & 2 & $65,6 x$ & 50,00 & $z$ & $40, \infty 0$ & 50,00 \\
\hline & 2 & $25,00 \mid$ & 8,70 & 0 & - & - & 2 & $40, \infty 0$ & 100,00 \\
\hline & 2 & 25,00 & 6,90 & 1 & 33,3 & 50,00 & 1 & $20, \infty$ & 50,00 \\
\hline & 8 & 100,00 & 5,05 & 3 & 100,00 & 37,50 & 5 & 100,00 & 62,50 \\
\hline & 10 & - & - & $\stackrel{0}{2}$ & - & - & 0 & - & - \\
\hline
\end{tabular}
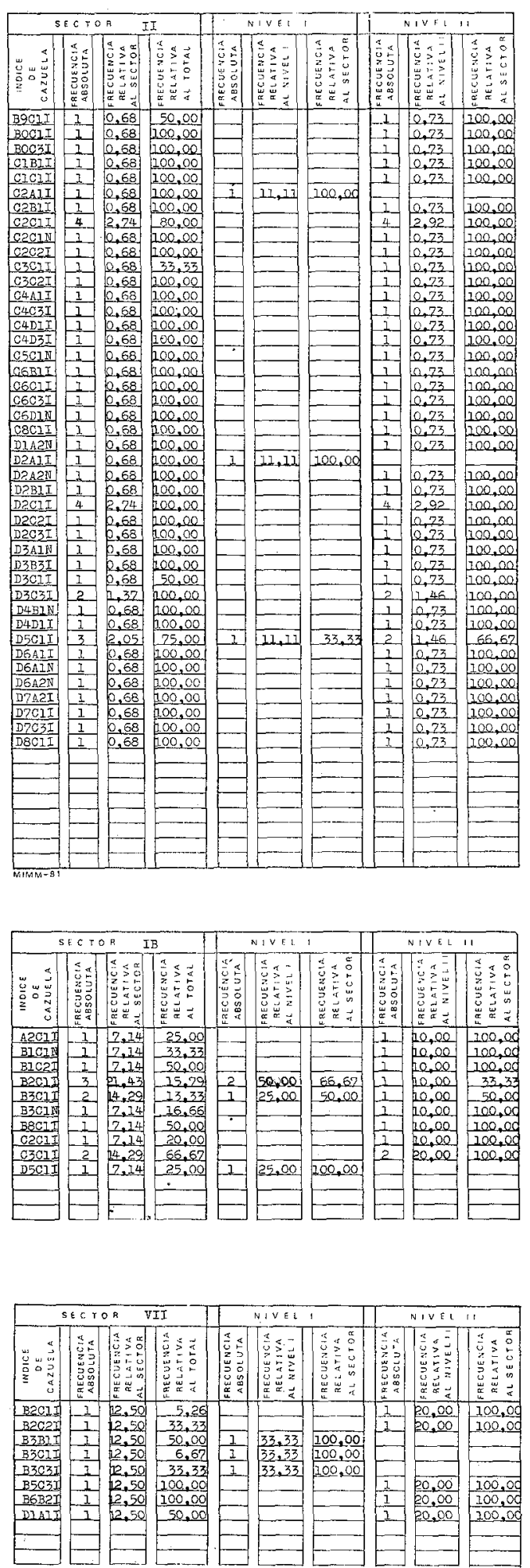

Tabla 4 Listado de frecuencia de variables y variantes por sectores $\mathrm{y}$ niveles. 


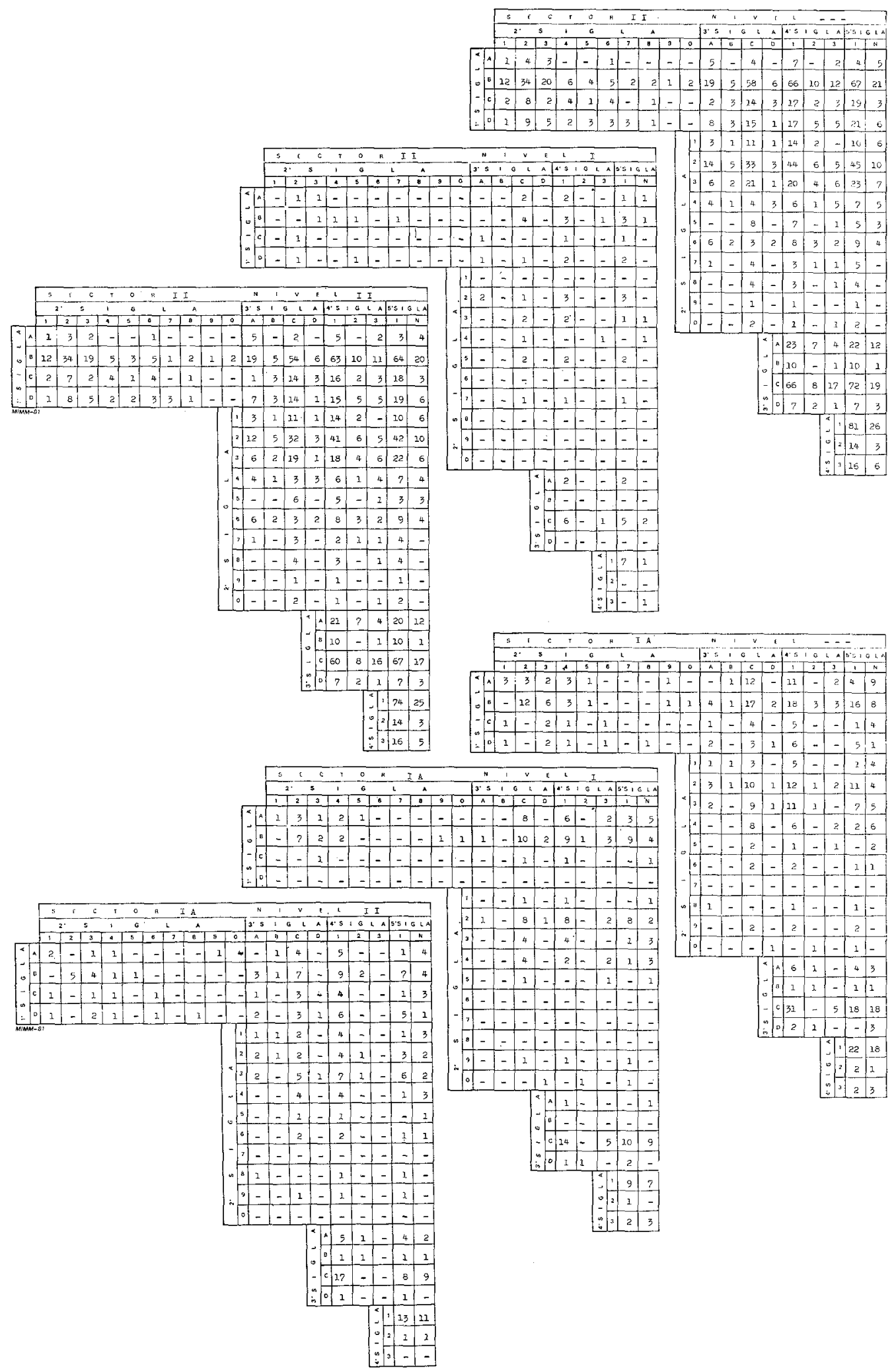

Tabla 5 Gráfico de combinación en frecuencia absoluta de las variables (Sectores IA y II). 


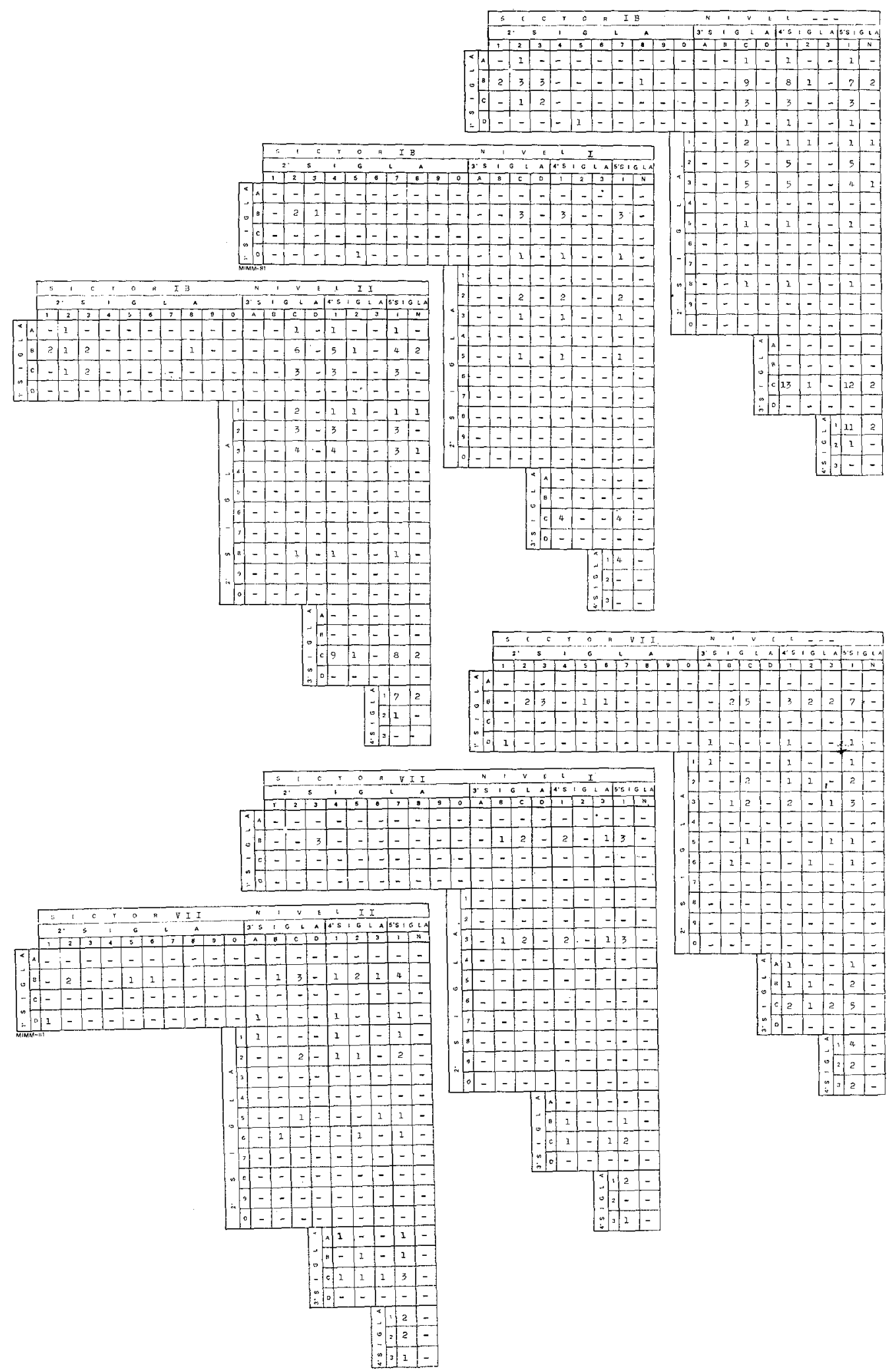

Tabla 6 Gráfico de combinaciones en frecuencia absoluta de las variables (Sectores IB y VII). 